\title{
Nutrition therapy with high intensity interval training to improve prostate cancer-related fatigue in men on androgen deprivation therapy: a study protocol
}

\author{
Brenton J. Baguley ${ }^{1 *}$, Tina L. Skinner ${ }^{1}$, Michael D. Leveritt ${ }^{1}$ and Olivia R. L. Wright ${ }^{1,2}$
}

\begin{abstract}
Background: Cancer-related fatigue is one of the most prevalent, prolonged and distressing side effects of prostate cancer treatment with androgen deprivation therapy. Preliminary evidence suggests natural therapies such as nutrition therapy and structured exercise prescription can reduce symptoms of cancer-related fatigue. Men appear to change their habitual dietary patterns after prostate cancer diagnosis, yet prostate-specific dietary guidelines provide limited support for managing adverse side effects of treatment. The exercise literature has shown high intensity interval training can improve various aspects of health that are typically impaired with androgen deprivation therapy; however exercise at this intensity is yet to be conducted in men with prostate cancer. The purpose of this study is to examine the effects of nutrition therapy beyond the current healthy eating guidelines with high intensity interval training for managing cancer-related fatigue in men with prostate cancer treated with androgen deprivation therapy.
\end{abstract}

Methods/design: This is a two-arm randomized control trial of 116 men with prostate cancer and survivors treated with androgen deprivation therapy. Participants will be randomized to either the intervention group i.e. nutrition therapy and high intensity interval training, or usual care. The intervention group will receive 20 weeks of individualized nutrition therapy from an Accredited Practising Dietitian, and high intensity interval training (from weeks 12-20 of the intervention) from an Accredited Exercise Physiologist. The usual care group will maintain their standard treatment regimen over the 20 weeks. Both groups will undertake primary and secondary outcome testing at baseline, week 8 , 12, and 20; testing includes questionnaires of fatigue and quality of life, objective measures of body composition, muscular strength, cardiorespiratory fitness, biomarkers for disease progression, as well as dietary analysis. The primary outcomes for this trial are measures of fatigue and quality of life.

Discussion: This study is the first of its kind to determine the efficacy of nutrition therapy above the healthy eating guidelines and high intensity interval training for alleviating prostate-cancer related fatigue. If successful, nutrition therapy and high intensity interval training may be proposed as an effective therapy for managing cancer-related fatigue and improving quality of life in men during and after prostate cancer treatment.

Trial registration: Australian New Zealand Clinical Trials Registry ACTRN12615000512527. Trial registered on the 22/5/ 2015.

Keywords: Prostate cancer, Nutrition therapy, Exercise, Cancer related fatigue

\footnotetext{
* Correspondence: b.baguley1@uq.edu.au

'School of Human Movement and Nutrition Sciences, The University of

Queensland, Brisbane, Australia

Full list of author information is available at the end of the article
} 


\section{Background}

Prostate cancer has become the most significant major malignancy of men, severely impacting disease-specific morbidity and mortality [1-3]. Advances in treatment of the disease, particularly through the use of Androgen Deprivation Therapy (ADT; a primary and mainstay treatment of prostate cancer), has seen prostate cancer 5-year survival rate increase to 92\% [2]. Despite ADT's efficacy in disease control, the physiological alterations resulting from ADT have profound adverse effects, including increased fatigue $[4,5]$, metabolic risk [6-8], cardiovascular risk $[6,9]$, change in body composition (increased fat mass and decreased muscle mass) $[10,11]$, and reduced functional capacity [12]; the amalgamation of these side effects severely reduces quality of life [13-15]. Cancer-related fatigue (CRF) is a distressing, persistent, subjective sense of physical, emotional and/or cognitive tiredness or exhaustion related to cancer or cancer treatment that is not proportional to recent activity and interferes with usual functioning [16]. CRF is the most common adverse effect of ADT, with up to $74 \%$ of men treated with ADT experiencing symptoms of CRF [4]. Incidence of CRF can be seen as early as 12 weeks after treatment initiation, and may last for longer than 12 months post-treatment in men treated with ADT $[4,5,17]$. Given the widespread use of $\mathrm{ADT}$ in managing prostate cancer progression, many men are living with ADT-related side effects during the course of treatment, and longer into prostate cancer survivorship. Current management strategies of CRF are predominantly through pharmacological therapy $[16,18]$; yet more recently, natural therapies such as diet and exercise have demonstrated clinically significant reductions of CRF symptoms in men with prostate cancer treated with ADT $[19,20]$.

The benefits of aerobic exercise (performed at $65-80 \%$ age predicted maximum heart rate [APMHR] three times per week) and progressive resistance training (812 exercises, $2-4$ sets of $8-12$ repetitions performed 2-3 times per week) in isolation, or when prescribed together, are well established for improving CRF in men with prostate cancer [21-25]. Recently, there has been particular interest in the use of high intensity interval training $\left[85-95 \%\right.$ peak heart rate $\left(\mathrm{HR}_{\text {peak }}\right)$ interspersed with period of active recovery] for improving CRF, body composition, and quality of life in oncological populations for managing treatment-related side effects [26-28]. In colorectal cancer survivors, 12 supervised high intensity interval training sessions $(4 \times 4 \mathrm{~min}$ bouts of cycling at $85-95 \% \mathrm{HR}_{\text {peak }}$, interspersed with $3 \mathrm{~min}$ of active recovery at $50-70 \% \mathrm{HR}_{\text {peak }}$ ) performed over 4 weeks showed greater improvements in cardiorespiratory fitness and total body mass, when compared to 12 supervised moderate intensity exercise sessions (50 min of cycling at $50-70 \% \mathrm{HR}_{\text {peak }}$ ) [29]. Thus, high intensity interval training may provide an optimal exercise prescription for improving various aspects of health that are typically impaired with ADT; however exercise at this intensity is yet to be conducted in men with prostate cancer.

Combined nutrition therapy and exercise prescription has the potential to optimize management for CRF and other ADT related side effects. Aerobic exercise (55$80 \%$ APMHR) performed 2-3 times per week for 12weeks, with healthy eating group based seminars every 2 weeks, has demonstrated clinically significant improvements in CRF and quality of life in sedentary men treated with ADT $[19,20]$. In contrast individual nutrition advice to meet the United Kingdom Dietary guidelines [30] with 30 min per day of brisk walking for 24 weeks demonstrated no significant changes in CRF or quality of life compared to usual care [31]. Thus it appears CRF may be better managed with structured exercise prescription and concurrent healthy eating. Whilst Bourke and colleagues $[19,20]$ demonstrated significant improvements in CRF, the nutrition consults were group-based, which fails to consider individual dietary requirements and is not representative of standard dietetic practice. Thus the effects of tailored nutrition therapy beyond the healthy eating guidelines, with adjunctive structured exercise prescription on the burden of CRF remains to be elucidated.

Dietary manipulation has been identified to be an important lifestyle factor to alleviate ADT related side effects [32-34]; yet the efficacy of dietary interventions in isolation during treatment or into prostate cancer survivorship are limited for supporting the adverse side effects seen from ADT. Importantly, prostate cancerspecific dietary guidelines provide limited support and guidance for alleviating adverse treatment-related side effects, including CRF [35, 36]. In non-oncological populations, structured individualized nutrition therapy by an Accredited Practising Dietitian is recommended for adults who are overweight, obese, insulin resistant, and have altered lipid and triglyceride metabolism [37-39]; all notable adverse side effects from ADT. Yet the translation of this nutrition therapy in prostate cancer is yet to be elucidated. Recently, a Mediterranean-style diet pattern has been shown to improve metabolic and cardiovascular parameters in men at risk of prostate cancer [40]. Adherence to an anti-inflammatory properties of a Mediterranean diet have shown small reductions in hypertension (reduced systolic blood pressure; $\mathrm{SE}=-1.44 \mathrm{~mm} \mathrm{Hg}$ [95\% CI, - $2.88-0.01$ ]; and diastolic blood pressure; $\mathrm{SE}=-0.70 \mathrm{~mm} \mathrm{Hg}[95 \% \mathrm{CI},-1.34-0.07$ ] [41], and risk of type 2 diabetes $(\mathrm{RR}=0.93 ; 95 \% \mathrm{CI}, 0.89-0.98)$ [42]; thus showing plausible metabolic and cardiovascular effects on known ADT related side effects. Yet practical application of the Mediterranean-style diet pattern to men treated with ADT for management of CRF and improving quality of life is yet to be ellucidated. 
Literature to date has encompassed general healthy eating guidelines for the management of prostate cancer treated related side effects $[19,20,31]$, however general guidelines do not take into account the specific dietary requirements needed to manage the ADT-related side effects. Therefore, the role of specific individualized nutrition therapy tailored to alleviate the side effects of ADT, particularly CRF, and improve quality of life warrants investigation.

This study aims to:

1. Investigate whether 12-weeks of nutrition therapy, compared to 12 weeks of usual care, can improve prostate CRF and quality of life in men treated with ADT.

2. Assess the combined benefits of 20-weeks of nutrition therapy with 8 -weeks of high intensity exercise (weeks 12-20), compared to 20 weeks of usual care, on CRF and quality of life in men treated with ADT.

We hypothesized that 12-weeks of nutrition therapy, compared to 12 weeks of usual care, will improve CRF and quality of life in men with prostate cancer treated with ADT. It is further hypothesized a 20-week nutrition therapy intervention with 8 weeks of high intensity interval training, compared to 20 weeks of usual care, will improve CRF and quality of life in men with prostate cancer treated with ADT. Secondary measures of body composition, functional capacity, metabolic syndrome and biomarkers such as prostate specific antigen, insulin like growth factor [43]-1, IGF-2, IGF binding protein-3, interleukin [IL]-6, and IL-8), will be measured and analyzed between and within groups. We hypothesis nutrition therapy alone, and with high intensity exercise will improve body composition, functional capacity, and biomarkers of metabolic syndrome and prostate cancer progression.

\section{Methods/design}

The study is a two-arm randomized controlled trial design conducted at The University of Queensland School of Human Movement and Nutrition Sciences. All primary and secondary outcomes will be measured at baseline, week 8,12 , and 20 . A total of 116 men with prostate cancer treated with ADT ( $>3$ months) will be randomized to either a 20 -week nutrition therapy with 8 weeks of high intensity exercise (performed from weeks 12 to 20) or a control group of usual care. A person independent to the study will conceal participant allocation, after baseline testing using a random number generator into either group, with equal probability. The study will be guided by the CONSORT statement [44] (Fig. 1).

\section{Participant eligibility}

To be eligible to participate in this study, men must meet the following inclusion criteria: (a) aged $\geq 18$ years, (b) non-smoker, or have quit smoking for $\geq 3$ months, (c) a diagnosis of prostate cancer, and have been undertaking ADT for $\geq 3$ months, (d) body mass index of 18.5-34.9 kg/m $\mathrm{m}^{2}$ (i.e. normal weight, overweight or obese class I). Exclusion criteria include: (a) taking any supplements other than a single multivitamin, unless stated for medical purposes, (b) have any musculoskeletal, neurological, respiratory, metabolic or cardiovascular conditions that may prevent safe completion of the exercise demands of the study, as determined by a Urologist, (c) current infection, (d) bone metastases, (e) currently undertaking high intensity exercise i.e. $\geq 90 \%$ heart rate $\max \left(\mathrm{HR}_{\max }\right)$ or $\geq 7$ rating of perceived exertion (RPE; Borg's rating of perceived exertion scale, category scale $0-10$ ).

\section{Recruitment and informed consent}

Participants will be recruited from the Mater Adults Hospital, Brisbane, Australia; The University of Queensland, Australia; and the Cancer Council Queensland, Australia. Participants will also be recruited by way of media releases, presentations and advertisements in newsletters, newspapers, and noticeboards at The University of Queensland, Cancer Council Queensland, and the Prostate Cancer Foundation of Australia support groups. All participants will be complete a brief telephone screening questionnaire to verify eligibility. Eligible participants will be asked to seek guidance from their Urologist regarding participation in the study, and sign the consent form prior to participating in the study. Approval of the trial protocol was obtained from the Mater Research Ethics Committee (HREC/15/MHS/38) and The University of Queensland Human Research Ethics Committee (2015001245) prior to recruitment.

\section{Randomisation}

After baseline testing, participants will be randomly allocated to either the nutrition therapy with high intensity exercise or the standard care group using a 1:1 ratio. The randomisation process will be conducted by a computerized system using a random number generator, with equal probability, by a person external to the study.

\section{Measurements}

All measurements for primary and secondary outcomes will take place at baseline, week 8 , week 12 , and week 20 (see Table 1). A familiarization trial including the functional capacity and peak aerobic power $\left(\dot{\mathrm{VO}}_{2 \text { peak }}\right)$ tests will take place at least 7 days prior to baseline testing. Participants will be asked to refrain from vigorous exercise, caffeine, alcohol, food and beverages (except for water) for $12 \mathrm{~h}$ before all testing sessions. 


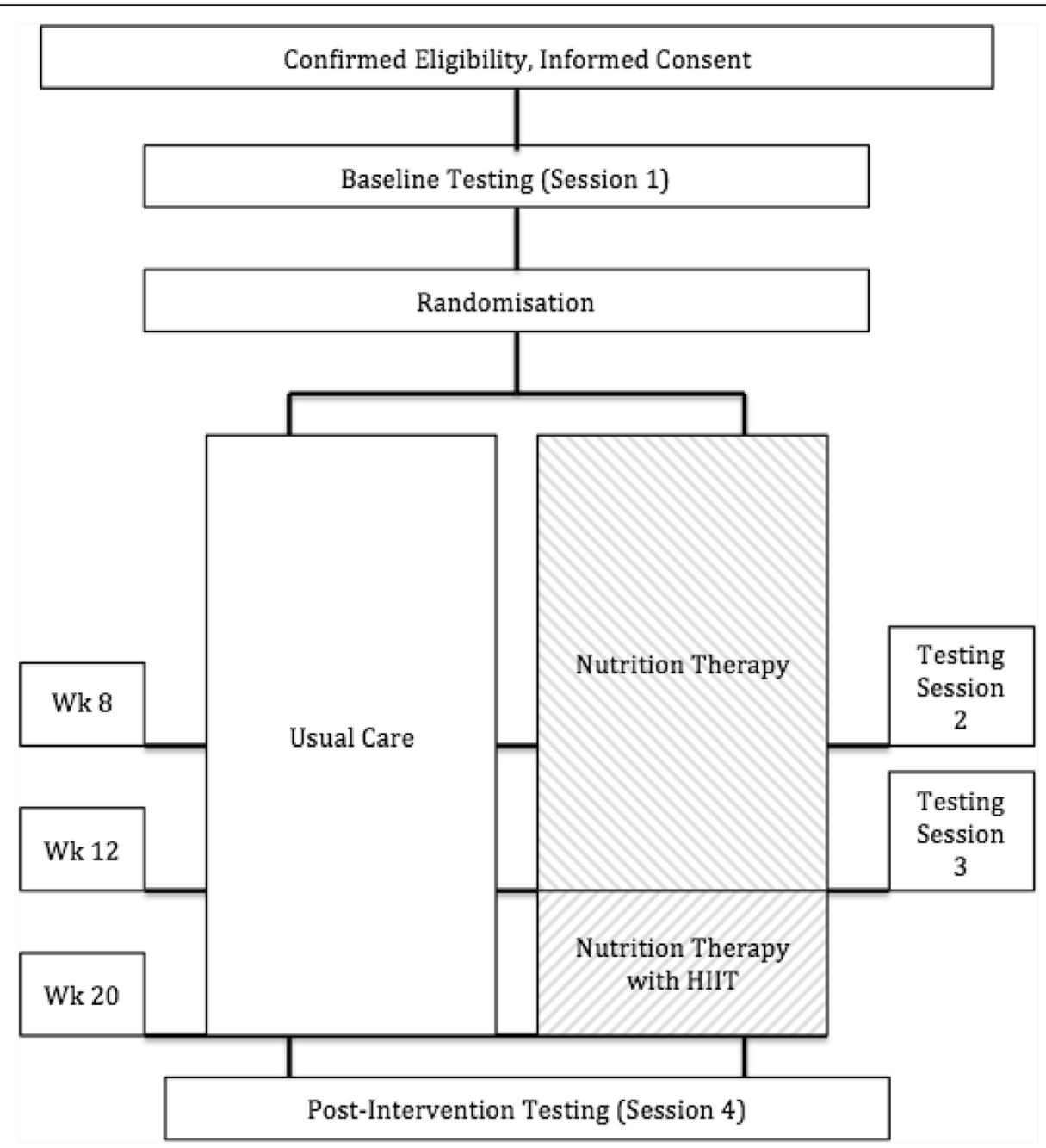

Fig. 1 CONSORT diagram and schematic representation of the study. Legend: Wk= week, HIIT = high intensity interval training

\section{Primary outcomes \\ Fatigue}

Fatigue will be measured using The Functional Assessment of Cancer Therapy: Fatigue (FACT-F) questionnaire, which reports weekly fatigue on a scale of $0-52$, with higher values indicating less fatigue [45]. The FACT-F has shown good reliability and validity (Cronbach's $\alpha$ : 0.93-0.94) for predicting clinically significant treatment outcomes of CRF [46].

\section{Cancer-specific quality of life}

The European Organization for Research and Treatment of Cancer (EORTC) QLQ-C30 is a 30 item cancerspecific instrument including the following domains; physical, role, emotional, social, and cognitive, global quality of life, fatigue, pain, and emesis. The EORTC QLQ-C30 has shown a high reliability (Cronbach's $\alpha$; $0.63-.80)$ in cancer patients [47].

\section{Prostate cancer-specific quality of life}

The EORTC QLQ-PR25 is a 25 question prostate cancer-specific instrument including domains; urinary, bowel, use of incontinence aids, treatment-related symptoms, sexual activity and sexual function [48]. The EORTC QLQ - PR25 questionnaire has shown good internal reliability (Cronbach's $\alpha$; 0.70-0.86) for prostate specific treatment-related side effects [49].

\section{General health and well-being}

The Medical Outcomes Study 36-Item Short-Form 36 Health Survey (SF-36) is scored by eight scales encompassing physical and mental measures [50]. The SF-36 questionnaire has widely been used to measure general health and well-being, with good construct validity (Cronbach's $\alpha ;>0.85$ ) in cancer patients [51]. 
Table 1 Data collection schedule

\begin{tabular}{|c|c|c|c|c|c|}
\hline \multirow[t]{2}{*}{ Assessments } & \multicolumn{5}{|c|}{ Time (weeks) } \\
\hline & Baseline $^{a}$ & & Week 8 & Week 12 & Week 20 \\
\hline \multicolumn{6}{|l|}{ Screening } \\
\hline Participant eligibility & $\checkmark$ & Randomization & & & \\
\hline Adult Pre-exercise Screening System & $\checkmark$ & & & & \\
\hline Medical history form and medications & $\checkmark$ & & & & \\
\hline \multicolumn{6}{|l|}{ Primary outcomes } \\
\hline Function Assessment of Cancer Therapy-Fatigue & $\checkmark$ & Randomization & $\checkmark$ & $\checkmark$ & $\checkmark$ \\
\hline European Organization of Research and Treatment of Cancer QLQ-C30* & $\checkmark$ & & $\checkmark$ & $\checkmark$ & $\checkmark$ \\
\hline European Organization of Research and Treatment of Cancer QLQ-PR25 & $\checkmark$ & & $\checkmark$ & $\checkmark$ & $\checkmark$ \\
\hline The Medical Outcomes Study 36-Item Short-Form 36 & $\checkmark$ & & $\checkmark$ & $\checkmark$ & $\checkmark$ \\
\hline \multicolumn{6}{|l|}{ Second Outcomes } \\
\hline Anthropometric physique traits & $\checkmark$ & Randomization & $\checkmark$ & $\checkmark$ & $\checkmark$ \\
\hline Body composition ${ }^{b}$ & $\checkmark$ & & $\checkmark$ & $\checkmark$ & $\checkmark$ \\
\hline Isometric strength & $\checkmark$ & & $\checkmark$ & $\checkmark$ & $\checkmark$ \\
\hline Chest and leg one repetition maximum test & $\checkmark$ & & $\checkmark$ & $\checkmark$ & $\checkmark$ \\
\hline Sit to stand test & $\checkmark$ & & $\checkmark$ & $\checkmark$ & $\checkmark$ \\
\hline Six-meter walk test & $\checkmark$ & & $\checkmark$ & $\checkmark$ & $\checkmark$ \\
\hline 400-meter walk test & $\checkmark$ & & $\checkmark$ & $\checkmark$ & $\checkmark$ \\
\hline Cardiorespiratory fitness $^{c}$ & $\checkmark$ & & $\checkmark$ & $\checkmark$ & $\checkmark$ \\
\hline Blood collection & $\checkmark$ & & $\checkmark$ & $\checkmark$ & $\checkmark$ \\
\hline Wollongong Dietary Inventory & $\checkmark$ & & $\checkmark$ & $\checkmark$ & $\checkmark$ \\
\hline 24-h diet recall ${ }^{d}$ & $\checkmark$ & & $\checkmark$ & $\checkmark$ & $\checkmark$ \\
\hline Depression Anxiety Stress Scale & $\checkmark$ & & $\checkmark$ & $\checkmark$ & $\checkmark$ \\
\hline Insomnia Severity Index & $\checkmark$ & & $\checkmark$ & $\checkmark$ & $\checkmark$ \\
\hline Physical Activity Enjoyment Scale & $\checkmark$ & & $\checkmark$ & $\checkmark$ & $\checkmark$ \\
\hline Godin Leisure-Time Physical Activity Questionnaire & $\checkmark$ & & $\checkmark$ & $\checkmark$ & $\checkmark$ \\
\hline Accelerometer ${ }^{\mathrm{e}, \mathrm{f}}$ & $\checkmark$ & & $\checkmark$ & $\checkmark$ & $\checkmark$ \\
\hline
\end{tabular}

${ }^{a}$ Nutrition and exercise intervention commences Week 0 after randomisation

${ }^{b}$ Dual energy X-ray absorptiometry

'ंO ${ }_{2 \text { peak }}$ test

${ }^{\mathrm{d} C}$ Conducted every 2 weeks

${ }^{\mathrm{e}}$ Actigraph GT3X+

to be worn for 7 consecutive days

*Cancer quality of life questionnaire for people with cancer

${ }^{\square}$ Cancer quality of life questionnaire for people with prostate cancer

\section{Secondary outcomes}

\section{Physique traits and body composition}

Height, body mass, and waist circumferences will be measured according to the International Society for the Advancement of Kinathropometry (ISAK) procedures [52].

Body composition (fat mass, lean mass, body fat percentage and bone mineral density) will be assessed using dual energy X-ray absorptiometry (DXA; Hologic Discovery A, Waltham, MA, USA).

\section{Muscular Strength and Power}

Isometric strength of dominate and non-dominate handgrip will be assessed using a spring-loaded grip dynamometer (TTM, Tokyo, Japan) to estimate physical performance [53], and muscular strength [54]. Participants will perform the test three times on each hand with the best result used for analysis. Muscular strength of the chest and legs will be measured using the one repetition maximum (1RM) chest and leg press methods, respectively [55]. The sit to stand test will be used to assess functional leg power [12]. Participants will perform the test three times with the best result used for analysis.

\section{Exercise capacity and cardiorespiratory fitness}

The six-meter walk test will involve participants walking a marked $10-\mathrm{m}$ distance as quickly as safely possible, 
with performance timed over the middle 6-m distance to minimize the influence of acceleration and deceleration [12]. The 400-m walk test will be used to estimate exercise capacity [12]; participants will be required to walk 10 laps out and back over a $20-\mathrm{m}$ course ( $400 \mathrm{~m}$ total) as fast as safely possible.

Cardiorespiratory fitness will be assessed using a $\dot{\mathrm{V}} \mathrm{O}_{2 \text { peak }}$ test. The test will involve a modified ramp protocol described by Wasserman et al [56] on a cycle ergometer. Participants will begin with 3 min of rest for respiratory normalization, followed by $4 \mathrm{~min}$ of warmup at a resistance of 50 Watt. The electrical resistance provided by the cycle ergometer increases incrementally by $20-30 \mathrm{~W} \cdot \mathrm{min}^{-1}$. Participants will cycle at a cadence between 60 and 70 revolutions per minute throughout the test. Heart rate will be continuously recorded throughout the exercise using a heart rate monitor (Polar FT1; Polar, Kempele, Finland) and blood pressure (Durashock Sphygmomanometer; Welch Allyn, New York, USA) will be recorded every 2 min throughout the test. At the conclusion of each minute participants will indicate their rating of perceived exertion (RPE) on the Borg 6-20 scale [57]. The test will be terminated when the participant reaches volitional fatigue or at the discretion of the researchers with consideration for exercise testing termination criteria as outlined by the American Association of Cardiovascular and Pulmonary Rehabilitation [58]. The gas analyzers and ventilometer will be calibrated prior to and verified after each test. Sampled expired air will be measured every $15 \mathrm{~s}$ using a turbine ventilometer (Morgan, Model 096, Kent, England). The ramp $\dot{\mathrm{V}} \mathrm{O}_{2 \text { peak }}$ protocol has good validity in comparison to the standard Bruce protocol [59]. $\dot{\mathrm{V}} \mathrm{O}_{2 \text { peak }}$ will be recorded as the highest $\dot{\mathrm{VO}}_{2}$ reading averaged over two consecutive readings.

\section{Blood collection and analysis}

A trained phlebotomist will extract, treat and subsequently store the blood at approximately $-80^{\circ} \mathrm{C}$ until later analysis. Analysis of blood samples through commercial ELISA kits (Thermo Fisher Scientific Australia Pty Ltd., Victoria, Australia; Randox Laboratories Ltd., West Virginia, USA; R\&D Systems Inc., Minneapolis, USA) will be used for PSA, IGF-1, IGF-2, IGFBP3, IL-6, IL-8, Hepcidin, total cholesterol, and triglycerides analysis.

\section{Dietary intake}

Participants will complete the Wollongong Dietary Inventory [60] (a comprehensive dietary history of intake over the past month) with cross-checking quantification from an Accredited Practising Dietitian. Narrative approaches to diet histories have been shown to provide good reproducibility and reliability compared to food records [61]. Food models and pictures of food portions (Great Ideas in
Nutrition, Coolangatta, Australia) will be utilized to improve the accuracy of food intake estimates [62].

\section{Other measures \\ Psychosocial: Depression Anxiety Stress Scale (DASS)}

The DASS is a 42-item self-report instrument design to measure the three related negative emotional states of depression, anxiety, and stress [63], and has been validated for measuring emotional states of depression (Cronbach's $\alpha: 0.94)$, anxiety (Cronbach's $\alpha: 0.88$ ) and stress (Cronbach's $\alpha:$ 0.93) in clinical populations [64].

\section{Insomnia Severity Index (ISI)}

The ISI questions relate to subjective qualities of the respondents sleep, including satisfaction with sleep patterns, the degree to which insomnia interferes with daily functioning, and how the respondent feels their insomnia is noticeable to others [65]. The ISI has shown excellent internal consistency (Cronbach's $\alpha$ : 0.90) for detecting symptoms of insomnia.

\section{Physical activity enjoyment scale (PACES)}

The physical activity enjoyment scale (PACES) will be used to assess participant enjoyment of the high intensity interval exercise PACES consists of 17 subscales that each relate to an aspect of enjoyment; along a 7-point continuum for each subscale, participants are asked to provide a rating to reflect their agreement with one of two bi-polar statements (e.g. 'I enjoy it' - 'I hate it') [66]. The PACES questionnaire has shown high internal consistency (Cronbach's $\alpha: 0.90)$ in measures of exercise enjoyment [67].

\section{Godin leisure-time physical activity questionnaire}

The Godin leisure-time physical activity questionnaire requires participants to recall during a typical 7-day week the frequency and duration of exercise completed at three separate intensities: mild, moderate, and strenuous intensity [68]. The Godin leisure-time physical activity questionnaire has shown high agreement and validation (agreement: 70.8\%) with minutes spent physically active, in comparison to an accelerometer in breast cancer survivors [69].

\section{Accelerometer}

Participants will be asked to maintain their current level of physical activity outside of the testing sessions for the duration of the study. Physical activity will be objectively assessed using the Actigraph GT3X+ accelerometer (Actigraph, Pensacola, Florida), a small, waist-worn, non-invasive device. Few studies have been published on the validity of the GT3X+ version of the Actigraph accelerometer specifically, however previous versions of the Actigraph accelerometer (CSA and GT1M) have demonstrated waist-worn validity in treadmill 
walking and running compared with indirect calorimetry $(r=0.56, p<0.001$ and $r=0.53, p<0.05$, respectively) in adults $[70,71]$.

\section{Interventions}

\section{Nutrition therapy with high intensity exercise}

Detailed diet histories will be conducted at baseline week 8,12 and 20 using the Wollongong Dietary Inventory [60], and $24 \mathrm{~h}$ diet recalls every two weeks during the intervention. Each consult will take approximately 30-45 min and involves an Accredited Practising Dietitian asking detailed questions about the foods consumed and the amount and frequency of consumption. The 20week diet emphasizes a total energy intake decrement using the Harris-Benedict predicted energy requirements [72]; with a dietary composition of $45-65 \%$ carbohydrate, $20-35 \%$ fat, saturated fat $<10 \%$ total energy intake, and $15-25 \%$ protein sources. Dietary advice will be tailored according to current body composition classification as 'normal, overweight or obese stage I' (see Fig. 2). A dietary energy reduction of 2000-4000 kJ/day will be emphasized at baseline consultation if body composition and diet intake are classified average or poor according to Fig. 2: Nutrition therapy schematic representation. The diet intervention will encompass a Mediterranean-style diet containing cruciferous vegetables (broccoli, bok choy, cauliflower) [73], tomato and tomato-paste containing products (lycopene) [74, 75], fruits, calcium and vitamin D containing foods, polyunsaturated, monounsaturated, omega 3 and 6 fatty acids $(<10-15 \%$ total energy intake) [76], and a reduced consumption of red meat ( $<2$ a week) [77, 78]; as seen in Table 2: Nutrition recommendations. Each individual nutritional consultation, will aim to make gradual changes to the participants' diets to best achieve a modified Mediterraneanstyle dietary pattern. Standard dietetics practice will be used to assess and providing guidance on managing the nutritional impact symptoms from treatment (including broader intolerances/allergies).

Each participant in the intervention group will be provided with a nutrition pamphlet for supportive education during the study to represent standard dietetic practice. The nutrition pamphlet will be provided at the first dietetic consult and is designed to facilitate the dietetic consultation to help maintain a Mediterranean-style diet pattern. The pamphlet consists of background information about nutrition requirements, optimal nutrition during prostate cancer based on current evidence

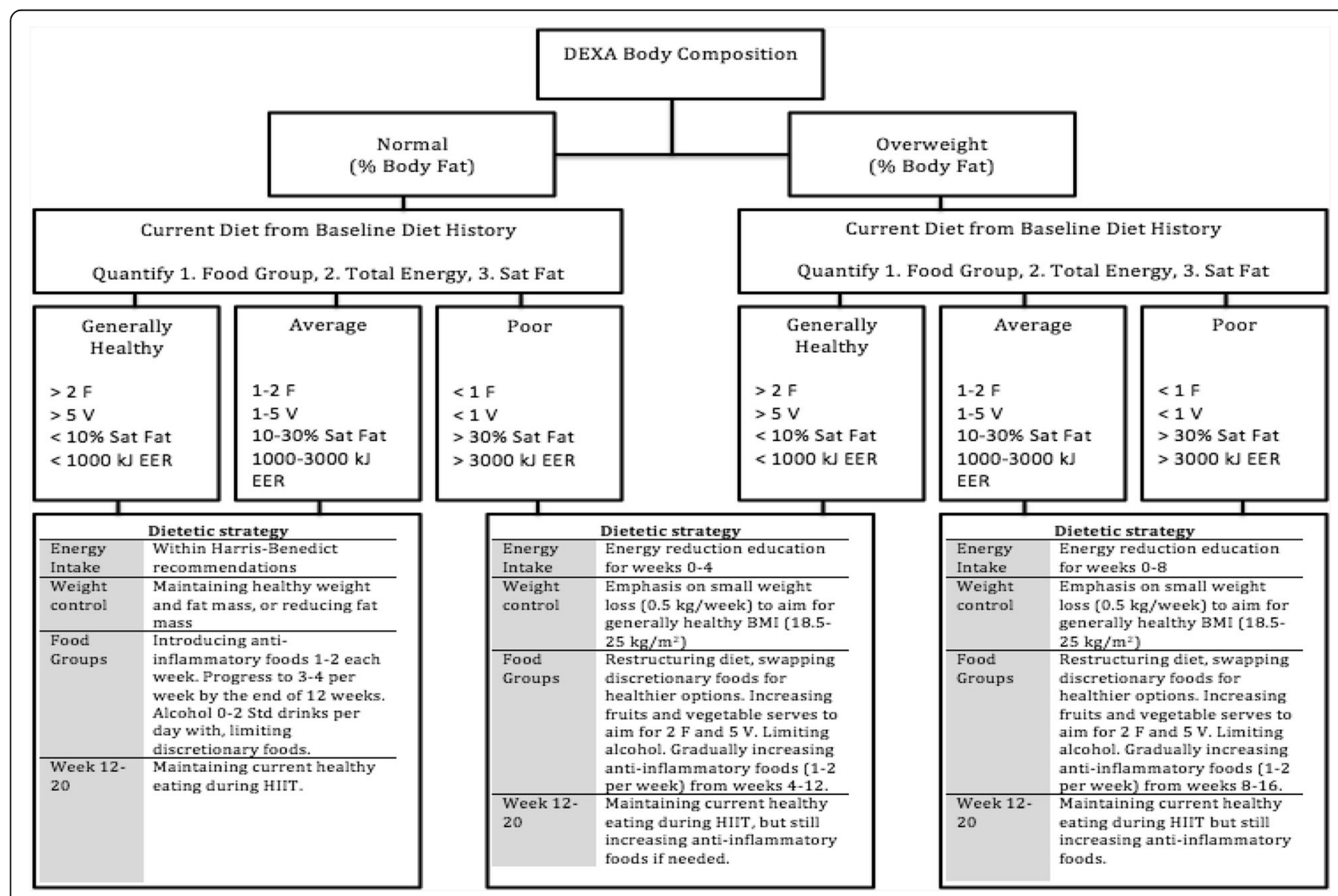

Fig. 2 Nutrition therapy assessment schematic representation. Legend: DEXA = Dual-energy $X$-ray absorptiometry, $F=$ fruit, $V=$ vegetable, Sat fat $=$ saturated fat, EER = estimated energy requirement, $\mathrm{HIIT}=$ high intensity interval training, Std = standard drink, BMI = body mass index 
Table 2 Nutrition recommendations

\begin{tabular}{|c|c|c|}
\hline Food/Nutrient & Serving summary & Recommendation \\
\hline Total Energy Intake & $\begin{array}{l}\text { As specified by your Accredited Practising Dietitian, } \\
\text { using the Harris-Benedict equation. }\end{array}$ & Within requirements \\
\hline Saturated fat & $\begin{array}{l}1 \text { serve = } 1 \text { tablespoon of butter, } 1 \text { sweet biscuit. } \\
\text { Excess fat on meat, full-cream dairy, take-away } \\
\text { foods, cakes, bakery items, processed meats, } \\
\text { vegetable oil. }\end{array}$ & $<10 \%$ total energy \\
\hline Refined Carbohydrates & $\begin{array}{l}\text { Serving size }=1(40 \mathrm{~g}) \text { Donut, } 30 \mathrm{~g} \text { potato chips, } \\
12 \text { hot chips, } 1 \text { can of soft drink }(375 \mathrm{ml}), 1 / 2 \\
\text { small chocolate bar }(25 \mathrm{~g})\end{array}$ & Limit consumption \\
\hline Whole Grains & 1 serve $=1$ slice of multigrain bread, $1 / 2$ cup oats, & 4-6 serves per day \\
\hline Fruits and Vegetables & $\begin{array}{l}\text { One serving }=1 \text { medium piece (apple, banana, } \\
\text { orange, pear), } 2 \text { small pieces (e.g. apricot, kiwifruit, } \\
\text { plums), } 1 / 2 \text { cup of tomato, } 1 / 2 \text { cup or carrot, } 1 / 2 \text { cup } \\
\text { green beans, } 1 / 2 \text { cup of pumpkin, } 100 \mathrm{~g} \text { mixed } \\
\text { frozen vegetables }\end{array}$ & 2 fruits and 5 vegetables per day \\
\hline Fibre & $\begin{array}{l}\text { Aim for } 30 \mathrm{~g} / \mathrm{day} \text { through your grains, vegetables, } \\
\text { and fruit intake }\end{array}$ & $\begin{array}{l}30 \mathrm{~g} / \text { day through grains, fruits } \\
\text { and vegetable intake }\end{array}$ \\
\hline Lentils/Beans & $\begin{array}{l}1 \text { cup per day of cooked or canned legumes/beans } \\
\text { such as lentil, chick peas or split peas }\end{array}$ & 1 cup per day \\
\hline Lean Meats & $\begin{array}{l}\text { Lean meats 3-4 times per week. Serving size of } 65 \mathrm{~g} \\
\text { include beef, lamb, veal, port, and } 80 \mathrm{~g} \text { for chicken, } \\
\text { turkey, duck, and } 2 \text { large eggs }\end{array}$ & Lean meats 3-4 times per week \\
\hline Meats with carcinogenic properties & $\begin{array}{l}\text { Red charred meat, visible from over cooking } \\
\text { or burning the mean. Processed meats } \\
\text { (i.e. salami, sausage) }\end{array}$ & Reduce or eliminate \\
\hline Oily Fish & $100 \mathrm{~g}$ per serve of fresh salmon, tuna, trout & 2-3 times per week \\
\hline Dairy & 1 cup of fresh, reduced fat milk, or soy milk & 2-3 serves per day \\
\hline Nuts and Seeds & $\begin{array}{l}30 \mathrm{~g} \text { (handful) of almonds, cashew nuts, walnuts, } \\
\text { macadamia nuts, brazil nuts, pumpkin seed, } \\
\text { sunflower seeds }\end{array}$ & $30 \mathrm{~g}$ per day \\
\hline Alcohol and discretionary foods & $\begin{array}{l}1 \text { standard drink of alcohol }=375 \mathrm{ml} \text { mid strength } \\
\text { beer, } 1 \text { glass of wine, } 30 \mathrm{ml} \text { spirit. Discretionary } \\
\text { foods = biscuits }\end{array}$ & $\begin{array}{l}0-2 \text { standard serves of alcohol, and } \\
\text { reduce or eliminate discretionary foods. }\end{array}$ \\
\hline
\end{tabular}

(kilojoule/cal, fatty acids, carbohydrate, meats and protein, fruits and vegetables, cruciferous vegetables, lycopene, soy isoflavones, vitamin $\mathrm{C}$ and calcium, alcohol and discretionary foods) and exercise during prostate cancer treatment. The pamphlet further consists of Decision Balance Tools to help change dietary patterns if needed throughout the dietetic consults by identifying possible barriers to adopting the nutrition therapy. These form part of standard dietetics practice involving motivational interviewing.

Baseline body composition scans (DXA) will classify participants as normal, overweight, or obese state I, according to age specific normative $\mathrm{Z}$ values for percentage of body mass index and fat mass [79]. The current diet will be quantified into food groups, total energy intake, and saturated fat intake. From this the diet will be classified as 'generally healthy', 'average' or 'poor' compared to the Australian Dietary Guidelines [80] (as seen in Fig. 2: Nutrition therapy schematic representation). To improve adherence, the nutrition therapy will not be prescriptive, and instead utilize individualized foodbased goals with the aim to progressively increase the intake of plant-based foods over the duration of the intervention to meet a modified Mediterranean-style diet pattern.

From weeks 12-20 participants will be required to visit The University of Queensland's School of Human Movement and Nutrition Sciences three times per week to complete high intensity interval training sessions. Prior to exercising, participants' heart rate and blood pressure will be measured for contraindications to commence exercising as outlined by the American College of Sports Medicine [81]. The high intensity exercise sessions will commence with $10 \mathrm{~min}$ of warm up at $50-70 \% \mathrm{HR}_{\text {peak }}$ before completing $4 \times 4$ min bouts of cycling at $85-95 \% \mathrm{HR}_{\text {peak. }}$ Each $4 \mathrm{~min}$ interval will be interspersed with a $3 \mathrm{~min}$ period of active recovery at $50-70 \% \mathrm{HR}_{\text {peak, }}$ totaling $38 \mathrm{~min}$ for the session. The high intensity exercise sessions will be conducted on an air- and magnetically-braked cycle ergometer (Wattbike 
Ltd., Nottingham, England). Participants will continue to receive the same dietetic intervention outlined above from weeks $12-20$.

\section{Usual Care}

Participants randomized to usual care will continue their usual medical care during this period. Participants in the usual care group will be monitored for 20 weeks and perform identical primary and secondary outcome measures at the same time points as outlined above for the intervention group.

\section{Sample Size Calculation}

Sample size calculations were complete using Vanderbilt Power and Sample Size software (Vanderbilt University, $\mathrm{TN})$. Combined diet and exercise interventions with CRF as an outcome are relatively limited. A diet and exercise intervention in men treated with ADT showed prostate cancer-specific CRF (as measured by FACT-F) scores improved by 3.7 - 14.2 (CI, 95\%; adjusted mean 8.9 points) in the intervention group compared to the control group [20]. Assuming FACT-F scores for this group were normally distributed with a SD of 8.9, a true difference between the experimental and control arms of 5.2 , and a power of 0.8 , we would need 47 experimental and control participants to be able to reject the null hypothesis. The type 1 error probability associated with this test of the null hypothesis is 0.05 . With an anticipated $20 \%$ attrition rate based on previous research from our laboratory, we would need 58 experimental and control group participants to be able to reject the null hypothesis.

\section{Statistical Analysis}

Data will be analyzed using the SPSS statistical software package (version 20.0, SPSS, Inc., Chicago, IL). Normality of the distribution for all outcome measures will be assessed using the Kolmogorov Smirnov test. Analyses will include standard descriptive statistics, t tests, correlation, regression and two-way repeated measures ANOVA or the comparable non-parametric test as necessary to examine differences between and within groups, at baseline and weeks 8,12 , and 20 .

\section{Data Collection, Management and Monitoring}

The principle investigator and trained research assistants will collect anthropometric, diet, exercise and lifestyle data from the study participants. Inter- and intra-tester reliability will be determined for data collecting investigators and research assistants for all outcome measures as appropriate. Computer files containing study data will be de-identified and password protected with access only available to study investigators. All research notes and data will be kept in a securely locked filing cabinet at
The University of Queensland School of Human Movement and Nutrition Sciences. The investigators will comply with the Good Clinical Practice (GCP) guidelines adopted by the Therapeutic Goods Administration and document all adverse events through the Human Research Ethics Committee (HREC). The study investigators will permit study-related monitoring, audits, and inspections by Mater HREC of all study related documents (e.g. source documents, regulatory documents, data collection instruments, study data) and study facilities (e.g. diagnostic laboratory). This study will be conducted in full conformance with the principles of the 'Declaration of Helsinki' according to international standards of GCP guidelines, applicable Australian government regulations and Institutional research policies and procedures.

\section{Discussion}

CRF is a distressing and prolonged symptom associated with prostate cancer treatment. Management of CRF is critical for improving quality of life during and after treatment for prostate cancer $[4,5,82]$. Nutrition therapy and exercise prescription have the potential to improve CRF and other prostate cancer disease- and treatment-related side effects [19-21, 25, 83-85]. This clinical trial is the first to investigate the efficacy of nutrition therapy above the healthy eating guidelines in managing prostate CRF. The lack of dietary guidelines for men with prostate cancer is a limitation in the current treatment and management of prostate cancer; thus investigating individual structured nutrition therapy provides novel insight to the efficacy of dietary modification in managing CRF and other side effects from ADT. This project will expand the exercise oncology literature by investigating the efficacy of high intensity interval training for reducing CRF and improving quality of life in men with prostate cancer. Considering both individual nutrition therapy beyond general healthy eating guidelines and high intensity interval training are yet to be investigated in men with prostate cancer, this landmark study will provide novel evidence to support the development of guidelines to optimise the management of CRF and other side effects from ADT. It is hypothesized that nutrition therapy alone, and with high intensity interval training will improve CRF and ADT-related side effects.

Dissemination of the results from this study to oncologists, urologists, prostate cancer nurses, dietitians, nutritionists, exercise physiologists and other exercise specialists will be important to ensure an evidence-based approach to the use of nutrition and exercise in the management of prostate CRF. Currently CRF is managed primarily with medications. Natural therapies such as diet and exercise provide a multi-faceted approach to 
managing CRF, which may in turn improve other associated side effects seen from ADT (e.g. metabolic and cardiovascular risk). With the rising incidence of prostate cancer and increasing survival rates, the primary outcome from this study will be to provide evidence to enhance clinical practice in nutrition, diet and exercise therapy to improve the lives of men suffering from the disease- and treatment-related side effects of prostate cancer.

\section{Abbreviations \\ ADT: Androgen deprivation therapy; APMHR: Age predicted maximum heart rate; CRF: Cancer related fatigue; DASS: Depression Anxiety Stress Scale; DXA: Dual energy X-ray absorptiometry; EORTC QLQ-C30: European Organization for Research and Treatment of Cancer quality of life questionnaire for people with cancer; EORTC QLQ-PR25: European Organization for Research and Treatment of Cancer prostate cancer specific quality of life questionnaire for people with cancer; FACT-F: The Functional Assessment of Cancer Therapy: Fatigue; $\mathrm{HR}_{\text {peak: }}$ : Heart rate peak; IGF: Insulin-like growth factor; LL: Interleukin; ISAK: International Society for the Advancement of Kinathropometry; ISI: Insomnia Severity Index; PACES: Physical activity enjoyment scale; RM: Repetition maximum; RPE: Rating of perceived exertion; SF-36: Short form 36 health survey; $\mathrm{VO}_{2 \text { peak: }}$ : Peak volume of oxygen consumption}

\section{Acknowledgements}

No other contributions are considered.

\section{Funding}

This study is funded by a Prostate Cancer Care Grant from Sanofi Aventis, through the Clinical Oncological Society of Australia.

\section{Availability of data and material}

Once data is obtained it will be made available after analysis and publication.

\section{Authors' contributions}

$\mathrm{BB}, \mathrm{TS}$, and $\mathrm{OW}$ are the trial coordinators who developed the study concept and protocols. ML assisted in further development of the project. Primary investigator BB will implement the protocol with $\mathrm{TS}, \mathrm{OW}$ and $\mathrm{ML}$ overseeing the collection and treatment of data. BB drafted the manuscript; all authors contributed to revision and approval of the final manuscript.

\section{Competing interests}

The authors declare that they have no competing interests.

\section{Consent for publication}

All eligible participants will be explained individual details will be de-identified and stored in a password-protected device only accessed by the research team Participants will be informed data collected will be intended for publication.

\section{Ethics approval and consent to participate}

Institutional Human Research Ethics Approval, The University of Queensland (2015001245), and The Mater Health Services Human Research Ethics Committee (HREC/15/MHS/38).

\section{Consent to participate}

All eligible participants will have the protocol, timelines, and outcome measures of the study explained to them. Participants will be required to gain medical consent from their General Practitioner and provide their signed and witnessed participant consent form prior to baseline testing. Participants will be informed that they are free to drop out of the study at any time without consequence, and that their data may still be used in the analysis unless otherwise specified in the withdrawal of consent form.

\section{Author details}

${ }^{1}$ School of Human Movement and Nutrition Sciences, The University of Queensland, Brisbane, Australia. ${ }^{2}$ Mater Research Institute, University of Queensland, Kent Street, Woolloongabba, Brisbane QLD 4102, Australia.
Received: 22 December 2015 Accepted: 17 December 2016

Published online: 03 January 2017

\section{References}

1. Australian Institute of Health and Welfare. Cancer in Australia: actual incidence and mortality data from 1982 to 2007 and projections to 2010. Asia Pac J Clin Oncol. 2011;7(4):325-38

2. Australian Institute of Health and Welfare: Cancer in Australia. Actua incidence data from 1991 to 2009 and mortality data from 1991 to 2010 with projections to 2012. Asia Pac J Clin Oncol. 2013;9:199-213.

3. Australian Institute of Health and Welfare. Cancer survival and prevalence in Australia: period estimates from 1982 to 2010. Asia Pac J Clin Oncol. 2013:9(1):29-39.

4. Langston B, Armes J, Levy A, Tidey E, Ream E. The prevalence and severity of fatigue in men with prostate cancer: a systematic review of the literature Support Care Cancer. 2013;21(6):1761-71.

5. Storey DJ, McLaren DB, Atkinson MA, Butcher I, Frew LC, Smyth JF, Sharpe M. Clinically relevant fatigue in men with hormone-sensitive prostate cancer on long-term androgen deprivation therapy. Ann Oncol. 2012;23(6):1542-9.

6. Keating NL, O'Malley AJ, Smith MR. Diabetes and cardiovascular disease during androgen deprivation therapy for prostate cancer. J Clin Oncol. 2006; 24(27):4448-56.

7. Kintzel PE, Chase SL, Schultz LM, O'Rourke TJ. Increased risk of metabolic syndrome, diabetes mellitus, and cardiovascular disease in men receiving androgen deprivation therapy for prostate cancer. Pharmacotherapy. 2008; 28(12):1511-22.

8. Milena B-B, Adrian SD, Denis CM, Michael AC, Majnu J, Josephine E, Shehzad B. Metabolic Syndrome in Men With Prostate Cancer Undergoing LongTerm Androgen-Deprivation Therapy. J Clin Oncol. 2006;24(24):3979-83.

9. Collier A, Ghosh S, McGlynn B, Hollins G. Prostate cancer, androgen deprivation therapy, obesity, the metabolic syndrome, type 2 diabetes, and cardiovascular disease: a review. Am J Clin Oncol. 2012;35(5):504-9.

10. Keogh JW, MacLeod RD. Body composition, physical fitness, functional performance, quality of life, and fatigue benefits of exercise for prostate cancer patients: a systematic review. J Pain Symptom Manag. 2012:43(1):96-110.

11. Haseen F, Murray LJ, Cardwell CR, O'Sullivan JM, Cantwell MM. The effect of androgen deprivation therapy on body composition in men with prostate cancer: Systematic review and meta-analysis. J Cancer Surviv. 2010;4(2):128-39.

12. Galvao DA, Taaffe DR, Spry N, Joseph D, Turner D, Newton RU. Reduced muscle strength and functional performance in men with prostate cancer undergoing androgen suppression: a comprehensive cross-sectional investigation. Prostate Cancer Prostatic Dis. 2009;12(2):198-203.

13. Drummond F, Kinnear H, O'Leary E, Donnelly, Gavin A, Sharp L. Long-term health-related quality of life of prostate cancer survivors varies by primary treatment. Results from the PiCTure (Prostate Cancer Treatment, your experience) study. J Cancer Surviv. 2015;1.

14. Dacal K, Sereika SM, Greenspan SL. Quality of life in prostate cancer patients taking androgen deprivation therapy. J Am Geriatr Soc. 2006;54(1):85-90.

15. Luo HC, Cheng LP, Cheng HH, Fu ZC, Liao SG, Li DS, Zheng WF, Lin GS, Zhu $J F$, Xu JF, et al. Long-term quality of life outcomes in patients with locally advanced prostate cancer after intensity-modulated radiotherapy combined with androgen deprivation. Med Oncol. 2014;31(6):991.

16. Berger AM, Abernethy AP, Atkinson A, Barsevick AM, Breitbart WS, Cella D, Cimprich B, Cleeland C, Eisenberger MA, Escalante CP, et al. Cancer-related fatigue. J Natl Compr Canc Netw. 2010;8(8):904-31.

17. Wang XS, Zhao F, Fisch MJ, O'Mara AM, Cella D, Mendoza TR, Cleeland CS. Prevalence and characteristics of moderate to severe fatigue: a multicenter study in cancer patients and survivors. Cancer. 2014;120(3):425-32.

18. Denlinger CS, Ligibel JA, Are M, Baker KS, Demark-Wahnefried W, Friedman DL, Goldman M, Jones L, King A, Ku GH, et al. Survivorship: fatigue, version 1.2014. J Natl Compr Canc Netw. 2014;12(6):876-87.

19. Bourke L, Doll H, Crank H, Daley A, Rosario D, Saxton JM. Lifestyle intervention in men with advanced prostate cancer receiving androgen suppression therapy: a feasibility study. Cancer Epidemiol Biomark Prev. 2011:20(4):647-57.

20. Bourke L, Gilbert S, Hooper R, Steed LA, Joshi M, Catto JWF, Saxton JM, Rosario DJ. Lifestyle Changes for Improving Disease-specific Quality of Life in Sedentary Men on Long-term Androgen-Deprivation Therapy for Advanced Prostate Cancer: A Randomised Controlled Trial. Eur Urol. 2014;65(5):865-72 
21. Segal RJ, Reid RD, Courneya KS, Sigal RJ, Kenny GP, Prud'Homme DG, Malone SC, Wells GA, Scott CG, Slovinec D'Angelo ME. Randomized controlled trial of resistance or aerobic exercise in men receiving radiation therapy for prostate cancer. J Clin Oncol. 2009;27(3):344-51.

22. Santa Mina D, Alibhai SM, Matthew AG, Guglietti CL, Pirbaglou M, Trachtenberg J, Ritvo P. A randomized trial of aerobic versus resistance exercise in prostate cancer survivors. J Aging Phys Act. 2013;21(4):455-78.

23. Galvao DA, Nosaka K, Taaffe DR, Spry N, Kristjanson L, McGuigan MR, Suzuki K, Yamaya K, Newton RU. Resistance training and reduction of treatment side effects in prostate cancer patients. Med Sci Sports Exerc. 2006;38(12):2045-52.

24. Culos-Reed SN, Robinson JW, Lau H, Stephenson L, Keats M, Norris S, Kline G, Faris P. Physical activity for men receiving androgen deprivation therapy for prostate cancer: benefits from a 16-week intervention. Support Care Cancer. 2010;18(5):591-9.

25. Cormie P, Galvão DA, Spry N, Joseph D, Chee R, Taaffe DR, Chambers SK, Newton RU. Can supervised exercise prevent treatment toxicity in patients with prostate cancer initiating androgen-deprivation therapy: A randomised controlled trial. BJU Int. 2014;115(2):256-66.

26. Edvardsen E, Skjonsberg OH, Holme I, Nordsletten L, Borchsenius F, Anderssen SA. High-intensity training following lung cancer surgery: a randomised controlled trial. Thorax. 2015;70(3):244-50.

27. Buffart LM, De Backer IC, Schep G, Vreugdenhil A, Brug J, Chinapaw MJ. Fatigue mediates the relationship between physical fitness and quality of life in cancer survivors. J Sci Med Sport. 2013;16(2):99-104.

28. Andersen C, Rorth M, Ejlertsen B, Stage M, Moller T, Midtgaard J, Quist M, Bloomquist K, Adamsen L. The effects of a six-week supervised multimodal exercise intervention during chemotherapy on cancer-related fatigue. Eur J Oncol Nurs. 2013;17(3):331-9.

29. Devin JL, Sax AT, Hughes Gl, Jenkins DG, Aitken JF, Chambers SK, Dunn JC, Bolam KA, Skinner TL. The influence of high-intensity compared with moderate-intensity exercise training on cardiorespiratory fitness and body composition in colorectal cancer survivors: a randomised controlled trial. J Cancer Surviv. 2015;10(3):467-79.

30. Food Standards Agency. Eat well, be well. https://www.food.gov.uk/sites/ default/files/multimedia/pdfs/publication/eatwell0708.pdf.

31. O'Neill RF, Haseen F, Murray LJ, O'Sullivan JM, Cantwell MM. A randomised controlled trial to evaluate the efficacy of a 6-month dietary and physical activity intervention for patients receiving androgen deprivation therapy for prostate cancer. J Cancer Surviv. 2015;9(3):431-40.

32. Saylor PJ, Keating NL, Smith MR. Prostate cancer survivorship: prevention and treatment of the adverse effects of androgen deprivation therapy. J Gen Intern Med. 2009:24 Suppl 2:S389-94.

33. Nguyen PL, Alibhai SMH, Basaria S, D'Amico AV, Kantoff PW, Keating NL, Penson DF, Rosario DJ, Tombal B, Smith MR. Adverse Effects of Androgen Deprivation Therapy and Strategies to Mitigate Them. Eur Urol. 2014;67(5):825-36.

34. Grossmann M, Zajac JD. Androgen deprivation therapy in men with prostate cancer: how should the side effects be monitored and treated? Clin Endocrinol (Oxf). 2011;74(3):289-93.

35. Rock CL, Doyle C, Demark-Wahnefried W, Meyerhardt J, Courneya KS, Schwartz AL, Bandera EV, Hamilton KK, Grant B, McCullough M, et al. Nutrition and physical activity guidelines for cancer survivors. CA Cancer J Clin. 2012;62(4):242-74.

36. Skolarus TA, Wolf AM, Erb NL, Brooks DD, Rivers BM, Underwood 3rd W, Salner AL, Zelefsky MJ, Aragon-Ching JB, Slovin SF, et al. American Cancer Society prostate cancer survivorship care guidelines. CA Cancer J Clin. 2014; 64(4):225-49.

37. Dietitians Associaion of Australia. DAA Best Practice Guidlelines for the Treatment of Overweight and Obesity in Adults. Canberra: Dietitians Association of Australia; 2012.

38. Dietitians Associaion of Australia. Evidence based practice guideliens for the nutritional management of type 2 diabetes mellitus for adults. Canberra: Dietitians Associaion of Australia; 2006.

39. National Heart Foundation of Australia. Dietary fats and dietary cholesterol for cardiovascular health. Canberra: National Heart Foundation of Australia; 2009

40. Ferris-Tortajada J, Berbel-Tornero O, Garcia-Castell J, Ortega-Garcia JA, Lopez-Andreu JA. Dietetic factors associated with prostate cancer: protective effects of Mediterranean diet. Actas Urol Esp. 2012;36(4):239-45.

41. Nissensohn M, Roman-Vinas B, Sanchez-Villegas A, Piscopo S, Serra-Majem L. The effect of the mediterranean diet on hypertension: a systematic review and meta-analysis. J Nutr Educ Behav. 2016;48(1):42-53.e41.
42. Schwingshackl L, Missbach B, Konig J, Hoffmann G. Adherence to a Mediterranean diet and risk of diabetes: a systematic review and meta-analysis. Public Health Nutr. 2015;18(7):1292-9.

43. van de Vijver LP, Kardinaal AF, Couet C, Aro A, Kafatos A, Steingrimsdottir L, Amorim Cruz JA, Moreiras O, Becker W, van Amelsvoort JM, et al. Association between trans fatty acid intake and cardiovascular risk factors in Europe: the TRANSFAIR study. Eur J Clin Nutr. 2000;54(2):126-35.

44. Moher D, Hopewell S, Schulz KF, Montori V, Gotzsche PC, Devereaux PJ, Elbourne D, Egger M, Altman DG. CONSORT 2010 explanation and elaboration: updated guidelines for reporting parallel group randomised trials. Int J Surg. 2012;10(1):28-55.

45. Yellen SB, Cella DF, Webster K, Blendowski C, Kaplan E. Measuring fatigue and other anemia-related symptoms with the Functional Assessment of Cancer Therapy (FACT) measurement system. J Pain Symptom Manag. 1997;13(2):63-74.

46. Cella D, Eton DT, Lai J-S, Peterman AH, Merkel DE. Combining Anchor and Distribution-Based Methods to Derive Minimal Clinically Important Differences on the Functional Assessment of Cancer Therapy (FACT) Anemia and Fatigue Scales. J Pain Symptom Manag. 2002;24(6):547-61.

47. Jocham HR, Widdershoven GAM, Dassen TWN, Halfens R. Reliability and validity of the EORTC QLQ-C30 in palliative care cancer patients. Cen Eur J Med. 2009;4(3):348-57.

48. Cella D, Nichol MB, Eton D, Nelson JB, Mulani P. Estimating Clinically Meaningful Changes for the Functional Assessment of Cancer Therapy_Prostate: Results from a Clinical Trial of Patients with Metastatic Hormone-Refractory Prostate Cancer. Value Health. 2009;12(1):124-9.

49. van Andel G, Bottomley A, Fossa SD, Efficace F, Coens C, Guerif S, Kynaston H, Gontero P, Thalmann G, Akdas A, et al. An international field study of the EORTC QLQ-PR25: a questionnaire for assessing the health-related quality of life of patients with prostate cancer. Eur J Cancer. 2008:44(16):2418-24.

50. Ware Jr JE, Sherbourne CD. The MOS 36-item short-form health survey (SF-36). I. Conceptual framework and item selection. Med Care. 1992;30(6):473-83.

51. Guirado VM, Taricco MA, Nobre MR, Couto Jr EB, Ribas ES, Meluzzi A, Brock RS, Dias MR, Rodrigues R, Teixeira MJ. Quality of life in adult intradural primary spinal tumors: 36-Item Short Form Health Survey correlation with McCormick and Aminoff-Logue scales. J Neurosurg Spine. 2013;19(6):721-35.

52. Stewart A, Marfell-Jones M, Olds T, H. DR. International Standards of Anthropometric Assessment. New Zealand: Internation Society for the Advancement of Kinathropometry; 2011.

53. Stevens PJ, Syddall HE, Patel HP, Martin HJ, Cooper C, Aihie Sayer A. Is grip strength a good marker of physical performance among communitydwelling older people? J Nutr Health Aging. 2012;16(9):769-74.

54. Cornish RS, Bolam KA, Skinner TL. Effect of Caffeine on Exercise Capacity and Function in Prostate Cancer Survivors. Med Sci Sports Exerc. 2015;47(3):468-75.

55. Taaffe DR, Duret C, Wheeler S, Marcus R. Once-weekly resistance exercise improves muscle strength and neuromuscular performance in older adults. J Am Geriatr Soc. 1999;47(10):1208-14.

56. Wasserman K, Hansen J, Sue D, Casaburi R, Whipp B. Principles of exercise testing and interpretiation: including pathophysiology and clinical appliactions. Philadelphia: Wolters Kluwer Health/Lippincott Williams \& Wilkins; 2012.

57. Borg GA. Psychophysical bases of perceived exertion. Med Sci Sports Exerc. 1982;14(5):377-81.

58. American Association of Cardiovascular Pulmonary Rehabilitation. Guidelines for cardiac rehabilitation and secondary prevention programs. Champaign, IL: Human Kinetics; 2004

59. Poole DC, Wilkerson DP, Jones AM. Validity of criteria for establishing maximal O2 uptake during ramp exercise tests. Eur J Appl Physiol. 2008;102(4):403-10.

60. Martin GS. The interviewer-administered, open-ended diet history method for assessing usual dietary intakes in clinical research: relative and criterion validation studies. Woollongong: The University of Woolongong; 2004.

61. Tapsell LC, Pettengell K, Denmeade SL. Assessment of a narrative approach to the diet history. Public Health Nutr. 1999;2(1):61-7.

62. Williams T. This = that: a life-size photo guide to food serves. Toowong, Qld: Trudy Williams Nutrition \& Dietetics; 2011.

63. Lovibond SH, Lovibond PF. Psychology Foundation of A: Manual for the depression anxiety stress scales. Sydney, N.S.W: Psychology Foundation of Australia; 1995. 
64. Nieuwenhuijsen K, de Boer AGEM, Verbeek JHAM, Blonk RWB, van Dijk FJH. The Depression Anxiety Stress Scales (DASS): detecting anxiety disorder and depression in employees absent from work because of mental health problems. Occup Environ Med. 2003;60 suppl 1:i77-82

65. Morin CM, Belleville G, Belanger L, Ivers $H$. The Insomnia Severity Index: psychometric indicators to detect insomnia cases and evaluate treatment response. Sleep. 2011;34(5):601-8.

66. Kendzierski DKD. Physical Activity Enjoyment Scale: Two validation studies. J Sport Exerc Psychol. 1991;13(1):50-64.

67. Latorre Roman PA, Garcia Pinillos F, Navarro Martinez AV, Izquierdo Rus T. Validity and reliability of Physical Activity Enjoyment Scale questionnaire (PACES) in children with asthma. J Asthma. 2014;51(6):633-8.

68. Godin G, Shephard RJ. A simple method to assess exercise behavior in the community. Can J Appl Sport Sci. 1985;10(3):141-6.

69. Amireault S, Godin G, Lacombe J, Sabiston C. Validation of the GodinShephard Leisure-Time Physical Activity Questionnaire classification coding system using accelerometer assessment among breast cancer survivors. J Cancer Surviv. 2015;9(3):532-40.

70. Abel MG, Hannon JC, Sell K, Lillie T, Conlin G, Anderson D. Validation of the Kenz Lifecorder EX and ActiGraph GT1M accelerometers for walking and running in adults. Appl Physiol Nutr Metab. 2008:33(6):1155-64.

71. Swartz AM, Strath SJ, Bassett Jr DR, O'Brien WL, King GA, Ainsworth BE. Estimation of energy expenditure using CSA accelerometers at hip and wrist sites. Med Sci Sports Exerc. 2000;32(9):S450-6.

72. Frankenfield DC, Muth ER, Rowe WA. The Harris-Benedict studies of human basal metabolism: history and limitations. J Am Diet Assoc. 1998;98(4):439-45.

73. Bosetti C, Filomeno M, Riso P, Polesel J, Levi F, Talamini R, Montella M, Negri E, Franceschi S, La Vecchia C. Cruciferous vegetables and cancer risk in a network of case-control studies. Ann Oncol. 2012;23(8):2198-203.

74. Etminan M, Takkouche B, Caamano-Isorna F. The role of tomato products and lycopene in the prevention of prostate cancer: a meta-analysis of observational studies. Cancer Epidemiol Biomarkers Prev. 2004;13(3):340-5.

75. Chen J, Song Y, Zhang L. Lycopene/tomato consumption and the risk of prostate cancer: a systematic review and meta-analysis of prospective studies. J Nutr Sci Vitaminol. 2013:59(3):213-23.

76. Pelser C, Mondul AM, Hollenbeck AR, Park Y. Dietary fat, fatty acids, and risk of prostate cancer in the NIH-AARP diet and health study. Cancer Epidemiol Biomarkers Prev. 2013;22(4):697-707.

77. Lee S-A, Zheng W. Well-Done Meat Intake, Heterocyclic Amine Exposure, and Cancer Risk. Nutr Cancer. 2009;61(4):437-46.

78. Mandair D, Rossi RE, Pericleous M, Whyand T, Caplin ME. Prostate cancer and the influence of dietary factors and supplements: a systematic review. Nutrition \& metabolism. 2014;11:30.

79. Kelly TL, Wilson KE, Heymsfield SB. Dual energy X-Ray absorptiometry body composition reference values from NHANES. PLOS ONE. 2009;4(9):e7038.

80. National Health and Medical Research Council. Australian dietary guidelines. Canberra: NHMRC; 2013. Available from: http://www.nhmrc.gov.au/_files nhmrc/publications/attachments/n55_australian_dietary_guidelines_130530. pdf.

81. American College of Sports Medicine. ACSM's new preparticipation health screening recommendations from ACSM's guidelines for exercise testing and prescription, ninth edition. Curr Sports Med Rep. 2013;12(4):215-7.

82. Köhler N, Gansera L, Holze S, Friedrich M, Rebmann U, Stolzenburg J-U, Truss M, Fahlenkamp D, Scholz H-J, Brähler E. Cancer-related fatigue in patients before and after radical prostatectomy. Results of a prospective multi-centre study. Support Care Cancer. 2014;22(11):2883-9.

83. Ornish D, Barnard RJ, Aronson WJ, McCormac P, McKnight DJ, Fein JD, Dnistrian AM, Weinstein J, Ngo TH, Mendell NR, et al. Intensive lifestyle changes may affect the progression of prostate cancer. J Urol. 2005;174(3): 1065-70.

84. Ornish D, Magbanua MJ, Weidner G, Weinberg V, Kemp C, Green C, Mattie MD, Marlin R, Simko J, Shinohara K, et al. Changes in prostate gene expression in men undergoing an intensive nutrition and lifestyle intervention. Proc Natl Acad Sci U S A. 2008;105(24):8369-74.

85. Galvão DA, Spry N, Denham J, Taaffe DR, Cormie P, Joseph D, Lamb DS, Chambers SK, Newton RU. A multicentre year-long randomised controlled trial of exercise training targeting physical functioning in men with prostate cancer previously treated with androgen suppression and radiation from TROG 03.04 radar. Eur Urol. 2014;65(5):856-64.

\section{Submit your next manuscript to BioMed Central and we will help you at every step:}

- We accept pre-submission inquiries

- Our selector tool helps you to find the most relevant journal

- We provide round the clock customer support

- Convenient online submission

- Thorough peer review

- Inclusion in PubMed and all major indexing services

- Maximum visibility for your research

Submit your manuscript at www.biomedcentral.com/submit
( Biomed Central 